regime used in our trial. However, it is worth noting that similarly high drop-out rates have been recorded in other well-regarded trials of antidepressant treatment. For example, in the National Institute of Mental Health trial, the drop-out rate from imipramine treatment was $33 \%$ over 18 months (Jacobson \& Hollon, 1996).

Dr Ogundipe's view about intention-totreat analysis is contrary to current statistical opinion and the recommendations of the British Medical Journal for the reporting of clinical trials (Altman, 1996; Schulz, 1996). The comment about baselines is not relevant here, since subjects who comply may fare differently and in an unpredictable way from those who do not comply. Thus, any observed differences between groups constructed in this manner may be due not to treatment but to factors associated with compliance. In this study, patients who dropped out were younger and had higher depression scores than those who completed the trial. The method of analysis should be consistent with the experimental design of a study. For randomised trials, such consistency requires the preservation of the random treatment assignment. Because methods that violate the principles of randomisation are susceptible to bias, they should not be used.

An analysis of the number needed to treat may be a sensible suggestion in general, although for a number of technical reasons it is not popular among statisticians (see Hutton, 2000). In any case, in this trial the high drop-out rate from the medication group would make the results of such an analysis suspect.

Marital discord was assessed using the Dyadic Adjustment Scale. As shown in Table 1 in the paper, the two treatment groups did not differ on this score, making it unlikely that this variable confounded the results.

Altman, D. G. (1996) Better reporting of randomised controlled trials: the CONSORT statement. British Medical Journal, 313, 570-57|.

Hutton, J. L. (2000) Number needed to treat: properties and problems. Journal of the Royal Statistical Society, Series A, 163, 403-419.

Jacobson, N. S. \& Hollon, S. D. (1996) Cognitivebehavior therapy versus pharmacotherapy: now that the jury's returned its verdict, it's time to present the rest of the evidence. Journal of Consulting and Clinical Psychology, 64, 74-80.

Schulz, K. F. (1996) Randomised trials, human nature, and reporting guidelines. Lancet, 348, 596-598.

J. Leff, B. Everitt Institute of Psychiatry, King's College London, Denmark Hill, London SE5 8AF

\section{Citalopram-induced bruxism}

There have been several reported incidents of iatrogenic bruxism (involuntary clenching or grinding of the teeth). These have involved diurnal bruxism (Micheli et al, 1993), felt to be associated with dopaminergic blockade, and nocturnal bruxism. Nocturnal bruxism has been reported with venlafaxine, a serotonin/noradrenaline reuptake inhibitor, which responded to gabapentin (Brown \& Hong, 1999), as well as three selective serotonin reuptake inhibitors (SSRIs), paroxetine (Romanelli et al, 1996), fluoxetine and setraline (Ellison \& Stanziani, 1993). In both reports the SSRI-associated bruxism was treated with buspirone.

I report two cases of nocturnal bruxism secondary to the SSRI citalopram, a previously unreported adverse effect. One patient was started on citalopram 20 $\mathrm{mg} /$ day. After 6 weeks the dose was increased to $40 \mathrm{mg}$. Ten days later nocturnal bruxism developed to such an extent that extraction of a molar was required. Buspirone was started and the bruxism ceased.

Another patient with panic disorder and moderate depression with somatic symptoms was referred to the clinic. The existing medication was a tricyclic and buspirone. Subsequent to non-response, medication was changed to citalopram, eventually reaching $40 \mathrm{mg} / \mathrm{day}$. After an improvement in mood a behavioural programme was used to treat his anxiety symptoms. Four months into the programme the buspirone was reduced from $10 \mathrm{mg}$ twice daily to none. Three weeks later he reported nocturnal bruxism. This ceased after reducing the citalopram to $20 \mathrm{mg} / \mathrm{day}$. Thus, in this case, occult nocturnal bruxism was revealed by the reduction of a treatment agent.

These cases highlight that nocturnal bruxism can occur in response to any of the SSRIs, and that induction may be dose-dependent. They add to the literature suggesting that nocturnal bruxism can be treated with buspirone.

Brown, E. S., Hong, S. C. (1999) Antidepressantinduced bruxism successfully treated with gabapentin. Journal of the American Dental Association, I30. 1467-1469.

Ellison, J. M. \& Stanziani, P. (1993) SSRI-associated nocturnal bruxism in four patients. Journal of Clinical Psychiatry, 54, 432-434.

Micheli, F., Fernandez Pardal, M., Gatto, M., et a (1993) Bruxism secondary to chronic antidopaminergic exposure. Clinical Neuropharmacology, 164, 315-323.
Romanelli, F., Adler, D. A. \& Bungay, K. M. (1996)

Possible paroxetine-induced bruxism. Annals of Pharmacotherapy, 301, 1246-1248.

M. E. J.Wise Paterson Centre for Mental Health, 20 South Wharf Road, London W2 IPD

\section{Psychological debriefing - does it never work?}

Mayou et al (2000) conclude in their 3-year follow-up study of road traffic accident victims that psychological debriefing is ineffective and has, in fact, adverse long-term effects. The intervention group reported significantly worse outcome at 3 years in terms of more severe psychiatric symptoms, impact of event symptoms, anxiety, depression, obsessive-compulsive problems and hostility, pain, major chronic health problems and financial problems. The findings support the suggestion that routine use of psychological debriefing among trauma victims should be discontinued (Bisson et al, 1997).

However, this conclusion is premature. A most serious problem in previous research is that the term psychological debriefing has been used for different types of interventions, for example, in terms of number of sessions and individual or group debriefing. Mayou et al offered individual one-session intervention, without any followup. This kind of intervention is contrary to most clinical thinking: first, assess the trauma; second, offer treatment accordingly. Nobody would recommend that all victims of traffic accidents should be given a standard surgical procedure of 15 minutes in the operating room. For patients with major traumas, the results may be worse than having no operation. The conclusion based on such an approach might easily be that surgery after traffic accidents should not be performed.

A flexible and individual approach is a much more reasonable and appropriate strategy (Rose et al, 1999). Future studies of psychological debriefing should use an individualised design including screening of psychopathology before intervention, if any, is offered. To assess the effect of one session of debriefing, only subjects who are likely to benefit from such a limited intervention should be included (i.e. those who are at greatest risk for post-traumatic stress disorder should be excluded).

The Impact of Event Scale scores for patients with high initial scores was $25.9 v$. 
11.8 in the control group (Mayou et al, 2000). This may indicate that the trauma of meeting a debriefer for 1 hour was comparable to the trauma of the traffic accident itself.

If the findings of Mayou et al are valid, it shows a tremendous potential for psychological intervention. However, we are still not convinced that a 1 -hour psychological intervention unintentionally can do so much harm.

Bisson, J. I., Jenkins, P. L., Alexander, J., et al (1997) Randomised controlled trial of psychological debriefing for victims of acute burn trauma. British journal of Psychiatry, I7I, 78-8I.

Mayou, R. A., Ehlers, A. \& Hobbs, M. (2000)

Psychological debriefing for road traffic accident victims. Three-year follow-up of a randomised controlled trial. British Journal of Psychiatry, 176, 589-593.

Rose, S., Brewin, C. R., Andrews, B., et al (1999) A randomized controlled trial of individual psychological debriefing for victims of violent crime. Psychological Medicine, 29, 793-799.

Ø. Ekeberg, E. Hem Department of Behavioural Sciences in Medicine, University of Oslo, PO Box IIII Blindern, N-0317 Oslo, Norway

\section{Diazepam in the treatment of GHB dependence}

We read with great interest the recent case reported by Price (2000) concerning the utility of diazepam in suppressing gammahydroxybutyrate (GHB) dependence and related withdrawal symptoms. Before describing the case Price states, "I believe this to be the first reported case of in-patient detoxification". However, the first report on the utility of the same doses of diazepam in treating GHB withdrawal syndrome was published a year earlier by our group in a patient taking about $181 \mathrm{~g} /$ day GHB for 4 months, for its euphoric and anxiolytic effects (Addolorato et al, 1999). On discontinuation of GHB, the patient showed a withdrawal syndrome consisting of high anxiety levels, tremor, sweating, tachycardia and nausea. Complete disappearance of drug withdrawal symptoms was achieved within 2 hours in the first day of treatment with diazepam $20 \mathrm{mg}$ orally administered, and the patient was treated with the same dose of diazepam for another 6 days. After suspension of the diazepam, the symptoms did not recur. Also in this case, the detoxification programme was safe. We are very pleased to know that our findings are in perfect agreement with that of Price; since several cases of GHB misuse and dependence have been described in recent years (see Addolorato et al, 2000), we think that these reported experiences, as well as the recent case described by Hutto et al (2000) about the utility of chloral hydrate, could be of clinical relevance, particularly considering the difficult management of these patients.

Addolorato, G., Caputo, F., Capristo, E., et al (1999) A case of gamma-hydroxybutyric acid withdrawal syndrome during alcohol addiction treatment: utility of diazepam administration. Clinical Neuropharmacology, 22 $60-62$.

_, _, _, et al (2000) Gamma-hydroxybutyric acid efficacy, potential abuse and dependence in the treatment of alcohol addiction. Alcohol, 20, 17-217-222.

Hutto, B., Faichild, A. \& Bright, R. (2000) Gammahydroxybutyrate withdrawal and chloral hydrate. American Journal of Psychiatry, 157, 1706.

Price, G. (2000) In-patient detoxification after GHB dependence (letter). British Journal of Psychiatry, 177, 181.

G. Addolorato, F. Caputo, E. Capristo,

G. Gasbarrini Institute of Internal Medicine, Catholic University of Rome, Largo A. Gemelli 8, 00168 Rome, Italy

\section{Seasonality of suicides}

We read with interest Yip et al's (2000) report on the decrease or disappearance in seasonal variation of suicides in the $1980 \mathrm{~s}$ and 1990s in England and Wales. The authors have suggested a similar trend in other countries (Ho et al, 1997; Yip et al, 1998). This paper inspired us to explore seasonality of suicide in Japan, where national data on monthly suicides are collected and published every year by the Ministry of Welfare. We examined monthly data by gender for the 15-year period 1982-1996. A daily mean suicide incidence was calculated for each month. Harmonic analysis (Pocock, 1974) was applied to identify seasonal components in the variation of monthly suicides.

A total of 332651 suicides (215 686 male, 116965 female) were identified for the period (mean suicide rate 25.8 and 13.2 per 100000 for men and women, respectively). During the entire period, the age distribution of suicide rates, which generally increases with age, and popular methods of suicides (approximately 60\% violent, nearly $35 \%$ non-violent, remainder unclassifiable or method not known) were generally unchanged. Regardless of gender, mean daily suicide incidence by month demonstrated a clear bimodal distribution, with a largest peak in April and a smaller peak in September. Harmonic analyses revealed that over $65 \%(66.7 \%$ for men and $66.2 \%$ for women) of the total variance in suicides was explained by the seasonal component, where one-cycle and two-cycle components illustrate the majority of total seasonal harmonics (one-cycle: $64.3 \%$ for men and $69.1 \%$ for women; two-cycle: $32.9 \%$ for men and $28.7 \%$ for women). No gender difference was found in the results from the harmonic analyses.

Unfortunately, no data on monthly suicides by age, by method or by place are available from the Japanese Government, which complicates further analyses regarding the backgrounds of such a marked seasonality in suicides. It may be noted that the school and business year begins in April in Japan, which may affect the seasonality of suicides, at least the largest peak of suicides in April. Overall, the results of our analyses indicate limitations in generalising from Yip et al's conclusions and suggest a wide variability in seasonality of suicide in different areas.

Ho, T. P., Chao, A. \& Yip, P. S. F. (1997) Seasona variation in suicides re-examined: no sex difference in Hong Kong and Taiwan. Acta Psychiatrica Scandinavica, 95, 25-31.

Pocock, S. J. (1974) Harmonic analysis applied to seasonal variations in sickness absence. Applied Statistics, 23, $103-120$

Yip, P. S. F., Chao, A. \& Ho, T. P. (1998) A reexamination of seasonal variation in suicides in Australia and New Zealand. Journal of Affective Disorders, 47. $|4|-150$.

\& Chiu, C. W. F. (2000) Seasonal variation in suicides: diminished or vanished. Experience from England and Wales, 1982-1996. British Journal of Psychiatry, 177, 366-369.

T. Sato Psychiatrische Klinik and Poliklinik, LMU München, Nussbraumstrasse 7, 80336 München, Germany

Reflecting on the diminished seasonal variation in suicides reported by Yip et al (2000), I was mindful of the recent extreme weather conditions - gales, floods, tornadoes - and the absence of 'Novemberness' - trees retaining their leaves, moderate temperatures, the absence of frost. Additionally in recent years we have been spared cold winters and lamented the loss of any recognisable British summertime. Therefore, one might wonder whether the disappearance of seasonal variation in suicide mirrors the 